Drimmelen, G. C. van \& Steyn, H. S. (1958). J. gen. Microbiol. 19, 324-329

\title{
The Keeping Quality of Freeze-Dried Brucella abortus Strain 19 Vaccine
}

\author{
BY G. C. vAN DRIMMELEN \\ Division of Veterinary Services, Onderstepoort, South Africa \\ AND H. S. STEYN \\ National Physical Research Laboratories, Pretoria, South Africa
}

SUMMARY: Concentrated Brucella abortus strain 19 vaccine is shown to deteriorate in time, even at low temperatures $\left(4-7^{\circ}\right)$. The loss of viability of vaccine sealed in pure nitrogen can be expressed as a coefficient. The value of this coefficient may be derived from the formula of a straight line fitting the means of the logarithms of the viability counts made 1 week to 6 months after freeze-drying.

Conventional liquid Brucella vaccine has poor keeping qualities because it consists of Brucella abortus strain 19 organisms which have to be fully viable to confer immunity on the inoculated animal. In the saline suspension of $60,000,000,000$ organisms $/ 5 \mathrm{ml}$. dose, which is the usual concentration of liquid vaccine, the viability of the organisms is progressively destroyed by light, heat and agitation during transport. The vaccine keeps fairly well for several weeks at $4^{\circ}$ and is best stored at this temperature, but transport at $4^{\circ}$ is rarely practicable, and oxidative processes destroy viability as soon as the temperature is raised. No chemical preservative can be used in this vaccine, as no toxoid or sporulating phase is involved, the immunizing quality being solely dependent on the high concentration of living bacteria.

Verwey \& Scheidy (1944) used the freeze-drying technique to maintain the viability of Brucella abortus strain 19 vaccine in an attempt to eliminate losses due to agitation and oxidation during transport and storage. Freeze-dried vaccine is, however, still susceptible to detrimental influences of heat and light (Naylor \& Smith, 1946). Alexander \& van Drimmelen (1956) freeze-dried this vaccine in highly concentrated form for economical distribution, the ampoules being sealed in purified nitrogen. Tests have proved this vaccine to have exceptional keeping qualities at room temperature (G. N. van Waveren et al. personal communication), but it is, of course, not superior to other freeze-dried Brucella vaccines when those are held at $4^{\circ}$.

All vaccines can be stored at this temperature for long periods, the loss of viability probably amounting to only a fraction of a logarithm of the viable content. However, some uncertainty was found to exist concerning the exact degree of deterioration in viability taking place under the most favourable storage conditions. As it was found desirable to stockpile Brucella vaccine at distribution points in South Africa as far as a thousand miles by rail from the producing laboratory, an accurate prediction of the viable count of stored 
freeze-dried vaccine became a necessity. The purpose of this report is to describe a method whereby the rate of loss of viability of different types of vaccine can be estimated.

\section{METHODS}

The vaccine used in this work was described previously by van Drimmelen (1956). It consisted of sodium carboxymethylcellulose-sedimented, concentrated Brucella abortus $\mathrm{S}$. 19, freeze-dried in $4 \mathrm{ml}$. amounts in $10 \mathrm{ml}$. ampoules and sealed in nitrogen. The number of micro-organisms surviving in freezedried material depends on the species and concentration of organisms, the suspending medium, and the method of freeze-drying and sealing of the containers. Where storage methods and freeze-drying techniques are constant, the production methods should be the only factors which influence the keeping quality of the product. Different types of vaccine were, therefore, produced, varying only in respect of viable count and freeze-drying mixtures, as follows :

Vaccine 1. Concentration of $160 \mathrm{ml}$. packed organisms/1. containing $0.5 \%$ $(w / v)$ ascorbic acid $+0.25 \%(w / v)$ thiourea (antioxidant).

Vaccine 2. $320 \mathrm{ml}$. packed organisms $/$. containing $0.5 \%(\mathrm{w} / \mathrm{v})$ ascorbic acid $+\mathbf{0} \cdot 25 \%(w / v)$ thiourea.

Vaccine 3. $160 \mathrm{ml}$. packed organisms/l. containing $1.8 \%(\mathrm{w} / \mathrm{v})$ ascorbic acid $+2 \cdot 8 \%(w / v)$ thiourea.

Vaccine 4. $320 \mathrm{ml}$. packed organisms $/$ l. containing $1.8 \%(\mathrm{w} / \mathrm{v})$ ascorbic acid $+2 \cdot 8 \%$ thiourea.

Counts were made of a number of viable organisms/ampoule from a representative number of batches of each vaccine, by the method of Miles \& Misra (1938) with minor modifications. These counts were made at selected time intervals during storage after freeze-drying, namely, immediately after drying and then after 1, 2, 3 weeks and 1, 3 and 6 months. The resultant figures were grouped for analysis in units of $10^{9}$ viable organisms/ampoule, i.e. the figure 2250 represents a count of $2250 \times 10^{9}$ viable organisms/ampoule.

To examine the distribution of viable counts at any particular time interval, a careful study was made of cases where more than thirty counts were available. As skew distributions were found on all occasions the cumulative frequencies were plotted on logarithmic probability paper and this resulted in approximately straight lines (Fig. 1), which indicated that the distribution was approximately log. normal. The observed counts are mean values multiplied by dilution factors which are not always constant; therefore, the distributions could not be considered as being Poisson distributions, which are known to occur frequently in such counting experiments.

$\chi^{2}$ tests were used to test the goodness of fit with the 'log. normal distribution' and gave satisfactory results. It was thus decided to use the logarithms of the observed counts (in thousand millions) for the selected periods, and to take them as being distributed normally.

The mean value of the logarithms of the counts was calculated for each period after freeze-drying, to find the trend in the deterioration of the 
logarithms. The 'standard deviations' and 'standard errors' were calculated independently for each of the distributions at the selected periods.

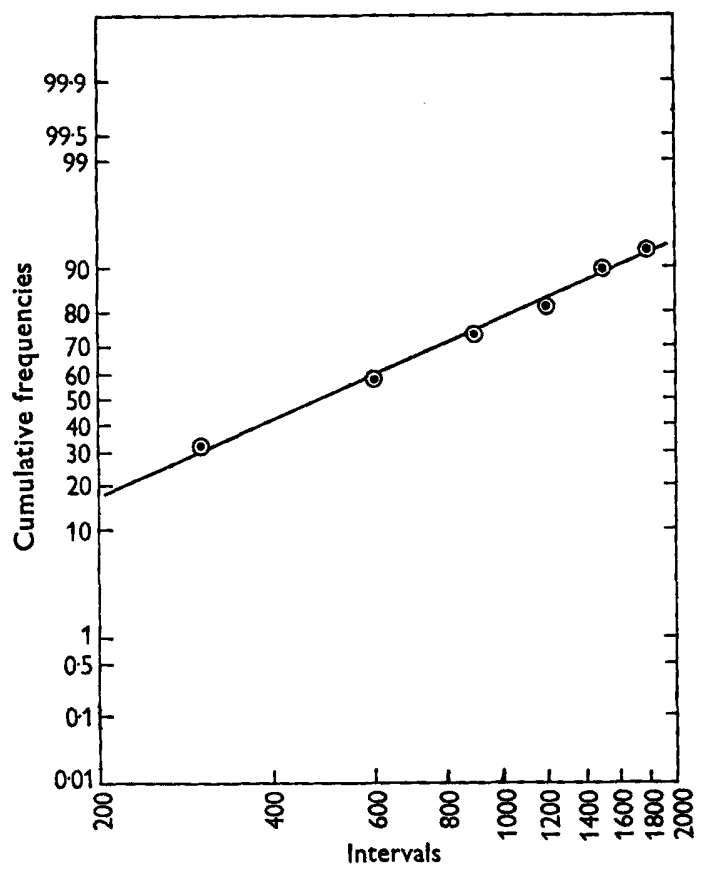

Fig. 1. Example showing that the relative cumulative frequencies of the viable counts when plotted on logarithmic probability paper give approximately a straight line. This indicates that the distribution at any particular time interval is approximately a log normal distribution.

\section{RESULTS}

The results are shown in Fig. 2 with the time periods, $x$ on the horizontal axis and the mean logarithms of the counts, $y$ on the vertical axis. The equations of the straight lines found to run through these mean values, obtained by the method of least squares, were as follows:
A. Vaccine $1 \quad y=2 \cdot 682-0 \cdot 012 x$.
Vaccine $2 y=2 \cdot 675-0 \cdot 018 x$.
$\begin{array}{rl}\text { B. Vaccine } 3 & y=3 \cdot 292-0 \cdot 017 x \text {. } \\ \text { Vaccine 4 } & y=3 \cdot 233-0 \cdot 017 x \text {. }\end{array}$

The results are illustrated in Fig. 2. Calculation of the standard error and standard deviation gives indications that the curves provide a method of characterizing the quality of the given types of Brucella vaccine by means of a coefficient derived from equations (1) to (4) above. The relations are found to be linear, which means that the rate of change of $y$ with $x$ remains constant, and is equal to the coefficient of $x$. The values of the coefficient of $x$ were: vaccine $1,0.012$; vaccine $2,0.018$; vaccine $3,0.017$; vaccine $4,0.017$. These coefficients show the average decrease in the mean of the logarithms of the counts per week. 
In Fig. 2 two limits are shown on each side of the mean for each time period. The outer limits are, respectively, at the points given by the mean + two standard deviations, and the mean - two standard deviations. These limits are such that only one out of twenty of the logarithms of the counts can be expected to fall outside them. For all practical purposes, therefore, the logarithms of all the counts at a particular time period may be taken as being between these two limits. The inner limits are respectively at points given by the mean + two standard errors and the mean - two standard errors. These are limits for the calculated mean values and such that only one out of twenty mean values from such samples can be expected to fall outside them.

As is indicated on the graphs on Fig. 2 the estimated mean values of the logarithms are never further than 2 standard errors from the straight lines. Since the standard deviations of the distributions of the logarithms were nearly always found to be smaller or equal to $\mathbf{0 . 4}$, it is suggested that for any mean value estimated from the graphs at any time period $x_{1}$ the maximum standard error may be taken as approximately equal to $0 \cdot 4 / \sqrt{ } n$. This should be interpreted as follows. If at this time period $x_{1} n$ counts were available, then the mean of the logarithms of the counts will not differ by more than $2 \times 0 \cdot 4 / \sqrt{ } n$ from the mean value on the graph. These values are specifically applicable to sodiumcarboxy-methyl-cellulose concentrated vaccine.

\section{DISCUSSION}

Statistical analysis of the results shows that the rate of loss of viability of any particular type of freeze-dried Brucella vaccine can be characterized by a constant which may be termed the coefficient of viability loss. The discovery of this constant is believed to be of practical value in organizing the distribution of vaccine in widespread areas, by making it possible to predict the viable count of vaccine stored at $4^{\circ}$ at distribution centres. Vaccine from the distribution centres could then be correctly labelled, as regards dosage, for periods of several months after leaving Onderstepoort. It is also a welcome discovery for use in studying the methods of production and freeze-drying, and should contribute to the replacement of empirical methods of freeze-drying by rational procedures.

It is clear from the values of the coefficient of $x$ that at the lower concentration of anti-oxidant $(0.5 \%$ ascorbic acid $+0.25 \%$ thiourea), the rate of viability loss was greater in the more concentrated vaccine (vaccine 2), i.e. 0.018 , as opposed to 0.012 in the less concentrated vaccine (vaccine 1 ), whereas at the higher concentration of anti-oxidant the rate of viability loss was the same in both concentrations of vaccine, i.e. 0.017 (vaccines 3 and 4). At the higher concentration of vaccine $(320 \mathrm{ml}$. packed organisms $/ \mathrm{l}$.) the rate of viability loss was greater in the vaccine containing the lower amount of anti-oxidant (vaccine 2), but at the lower concentration of vaccine (160 ml. packed organisms/l.), the rate of viability loss was greater in the vaccine containing the higher amount of anti-oxidant (vaccine 3). This indicates that excess anti-oxidant and insufficient anti-oxidant both adversely affected the 

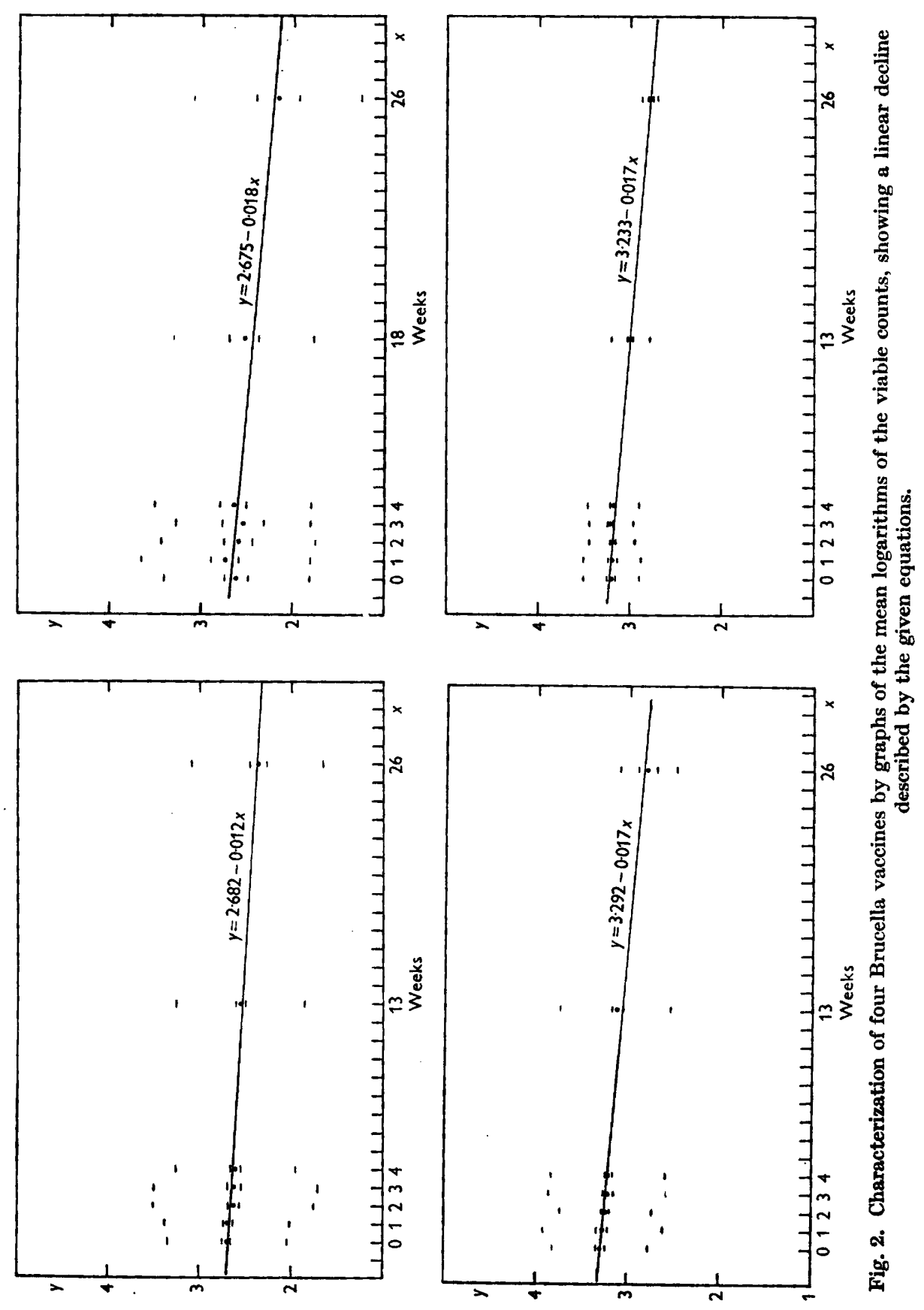
viability of the vaccine during storage, and shows that for maximum stability the anti-oxidant incorporated in the freeze-dried vaccine should be balanced with the concentration of viable organisms present.

It is known from experience that more concentrated vaccine requires higher anti-oxidant protection during freeze-drying than less concentrated vaccine, and evidence is available that excess of anti-oxidant during freeze-drying also is detrimental to the vaccine. This aspect is being analysed at present.

The present data show that an increase of anti-oxidant in freeze-drying mixtures for vaccines of $160 \mathrm{ml}$. packed organisms/l., although increasing the survival rate immediately after freeze-drying from a mean log. viable count of 2.682 to a mean of 3.292 (graphs 1 and 3), also results in an increased rate of deterioration during storage (coefficients of viability loss 0.012 and 0.017 ). For vaccines of $320 \mathrm{ml}$. packed organisms/l. an increase in anti-oxidant causes both an increase in immediate survival (values $2 \cdot 657$ and $3 \cdot 233$ graphs 2 and 4), and an increased stability during storage (coefficients 0.018 and 0.017). Based on data of this nature a system of distributing concentrated Brucella vaccine in bulk in refrigerated containers to advance centres of storage and distribution can be arranged. The vaccine can be labelled at the time of final issue, and the dosage calculated, in relation to the known rate of deterioration, by applying the correct formula for the type of vaccine used.

The director of Veterinary Services and the President of the South African Council for Scientific and Industrial Research are thanked for permission to publish this report. Technical assistance from Mr F. D. Horwell and Miss H. E. Smith at Onderstepoort, and Miss E. Z. Pretorius and Miss G. Dykstra of the C.S.I.R. is warmly appreciated.

\section{REFERENCES}

Alexander, R. A. \& van Drimmelen, G. C. (1956). The economy of immunizing cattle with freeze-dried Brucella vaccine. S. Afr. J. Sci. 52, 216.

Drimmelen, G. C. van (1956). Strain 19 Brucella Vaccine. II. The preparation of freeze-dried live vaccine. Onderstepoort J. vet. Sci. 27, 215.

Mrues, A. A. \& Misra, S. (1938). The estimation of the bactericidal powers of the blood. J. Hyg., Camb. 38, 732.

NAYLOR, H. B. \& Smith, P. A. (1946). Factors affecting the viability of Serratia marcescens during dehydration and storage. J. Bact. 52, 565.

Verwey, W. E. \& Scheidy, S. F. (1944)). The stability of Br. abortus vaccine desiccated by lyophilization. Proc. 48th Ann. Meet. U.S.L. San. Ass. p. 105. 